\title{
PRIMER REGISTRO DEL ÁGUILA BLANCA Y NEGRA Spizaetus melanoleucus EN LA RESERVA NACIONAL ALLPAHUAYO MISHANA, AL NORESTE DE LA AMAZONÍA PERUANA
}

\author{
María Isabel TORRES-VÁSQUEZ ${ }^{1}$, Juan DÍAZALVÁN , Jeder PIZANGO MURRIETA
}

1 Universidad Nacional de la Amazonía Peruana (UNAP), e-mail:neopelma@gmail.com; jdiazalvan@gmail.com

2 Reserva Nacional Allpahuayo Mishana-Servicio Nacional de Áreas Naturales Protegidas (RNAM-SERNANP)

\section{RESUMEN}

Reportamos la presencia del Águila Blanca y Negra Spizaetus melanoleucus en la Reserva Nacional Allpahuayo Mishana (RNAM) y en bosques de tierra firme en los alrededores del río Sucusari, ampliando su rango de distribución hacia el norte del río Amazonas y al mismo tiempo se incrementa a 498 el número de especies de aves para la RNAM. Describimos algunos detalles sobre el comportamiento de la especie y el estado de conservación de los bosques en donde fueron observados los individuos.

PALABRAS CLAVE: Accipitridae, Ampliación de Rango, Reserva Nacional Allpahuayo Mishana.

\section{FIRST RECORD OF THE BLACK AND WHITE HAWK EAGLE Spizaetus melanoleucus IN THE ALLPAHUAYO MISHANA NATIONAL RESERVE, NORTHEAST OF THE PERUVIAN AMAZON}

\section{ABSTRACT}

We report the presence of the Black-and-white Hawk-Eagle Spizaetus melanoleucus in the Allpahuayo Mishana National Reserve (AMNR) and in the terra firme forest around the Sucusari river, expanding its distribution range to the north of the Amazon river and at the same time increasing to 498 the number of bird species for the AMNR. We describe some details about the behavior of the species and the current state of conservation of the forests where the individuals were sighted.

KEYWORDS: Accipitridae, Allpahuayo Mishana National Reserve, Range extension. 


\section{INTRODUCCIÓN}

El Águila Blanca y Negra Spizaetus melanoleucus pertenece a la familia Accipitridae (Ferguson-Lees \& Christie, 2001) y tiene un rango de distribución muy amplio que abarca territorios de países como Ecuador, Colombia, Brasil, Venezuela, Bolivia y Argentina en el continente sudamericano, y desde el sur de Centro América hasta México (BirdLife International, 2017). A pesar de esto, es una especie poco común a lo largo de su rango de distribución. Spizaetus melanoleucus no se encuentra catalogada como amenazada, aunque su población está disminuyendo globalmente debido a la pérdida de hábitat y a la perturbación del hábitat ocasionada por el hombre (BirdLife 2017; IUCN, 2017).

En Perú, esta especie se encuentra distribuida al este de los Andes, desde el norte al sureste del país, en los departamentos de Loreto (principalmente al sur del río Amazonas), Ucayali, Madre de Dios, Amazonas, la zona este de San Martín, Huánuco, Cerro de Pasco, Junín y la zona norte de Cusco (Schulenberg et al., 2010; Wiley et al. 2017). Existen pocos registros de la especie y se conoce muy poco sobre su biología y comportamiento (Robinson, 1994; Adams \& Williams, 2017). En esta nota reportamos el registro del Águila Blanca y Negra Spizaetus melanoleucus en los bosques de la Reserva Nacional Allpahuayo Mishana (RNAM) y alrededores del río Sucusari, al mismo tiempo hacemos algunos apuntes sobre su comportamiento.

\section{ÁREA DE ESTUDIO Y MÉTODOS}

Los registros que se reportan corresponden a diversas exploraciones llevadas a cabo en distintos puntos dentro de la RNAM y en algunas áreas de los alrededores del río Sucusari, cuenca del río Napo, entre los años 1985 y 2017 (Tabla 1, Figura 1). Estos registros se llevaron a cabo de manera sistemática y oportunista, empleando binoculares para las detecciones visuales y una cámara Nikon Coolpix P900 con un lente de $2000 \mathrm{~mm}$ para las tomas fotográficas. Las coordenadas que se reportan fueron referenciadas en campo con un GPS. Las observaciones sobre el comportamiento fueron realizadas entre las 7:30 y 10:00 $\mathrm{h}$. Tres registros adicionales de la especie fueron tomadas del portal especializado de registros en línea de aves a nivel mundial eBird (www.ebird.org), de libre acceso.

\section{RESULTADOS}

La especie fue registrada en dos oportunidades por JDA y Noam Shany (12 y 14 de junio 2004), desde los puentes colgantes de la empresa Explornapo Lodge (distrito de Mazan, Provincia de Maynas), en la cuenca del río Sucusari, al este del río Napo, el individuo observado se encontraba perchado en la copa de un árbol a una altura aproximada de $35 \mathrm{~m}$ sobre el nivel del suelo, en un bosque primario sobre arcilla. Posteriormente JDA registró a Spizaetus melanoleucus el 10 de marzo y 26 de julio del 2013, en los alrededores de la comunidad de San Martin (distrito de San Juan, Provincia de Maynas), en la primera oportunidad se observó a un individuo solitario en vuelo cerca de la copa de los árboles, en un bosque inundable, próximos a la quebrada San Martín y en la segunda se observó a un individuo volando hacia el interior del bosque sobre tierra firme, desde una percha a una altura aproximada de $25 \mathrm{~m}$ cerca de un claro de bosque. Es probable que este individuo estuviera acechando a un grupo de monos frailes Saimiri macrodon que se encontraban en las inmediaciones.

Tabla 1. Lugares de registros de Spizaetus melanoleucus en el departamento de Loreto, Perú.

\begin{tabular}{|c|c|c|c|c|c|}
\hline $\mathbf{N}^{0}$ & Localidades & $\begin{array}{c}\text { Coordenadas } \\
\text { (UTM) }\end{array}$ & $\begin{array}{l}\text { Altitud } \\
(\mathrm{m})\end{array}$ & Autores & Fechas \\
\hline 1 & Explornapo Lodge, río Sucusari & 7314159639674 & 100 & Van Remsen* & $25 / 06 / 1985$ \\
\hline 2 & ACTS, río Sucusari & 7323999640625 & 108 & Rafael Campos-Ramírez* & 29/07/1998 \\
\hline 3 & Explornapo Lodge, río Sucusari & 7314159639674 & 99 & $\begin{array}{l}\text { Juan Díaz Alván \& } \\
\text { Noam Shany }\end{array}$ & $\begin{array}{l}12- \\
14 / 06 / 2004\end{array}$ \\
\hline 4 & Reserva Nacional Allpahuayo Mishana & $671396 \quad 9571717$ & 95 & Juan Díaz Alván & $10 / 03 / 2013$ \\
\hline 5 & Reserva Nacional Allpahuayo Mishana & 6716109570786 & 104 & Juan Díaz Alván & $26 / 07 / 2013$ \\
\hline 6 & Reserva Nacional Allpahuayo Mishana & 6760389563170 & 136 & María Isabel Torres Vásquez & $11 / 03 / 2014$ \\
\hline 7 & Reserva Nacional Allpahuayo Mishana & 6761619562647 & 127 & Thomas Valqui* & $22 / 10 / 2016$ \\
\hline 8 & Reserva Nacional Allpahuayo Mishana & 6673879570748 & 98 & $\begin{array}{l}\text { Jeder Pizango Murrieta \& } \\
\text { María Isabel Torres Vásquez }\end{array}$ & $12 / 03 / 2017$ \\
\hline
\end{tabular}

*Fuente: eBird. 


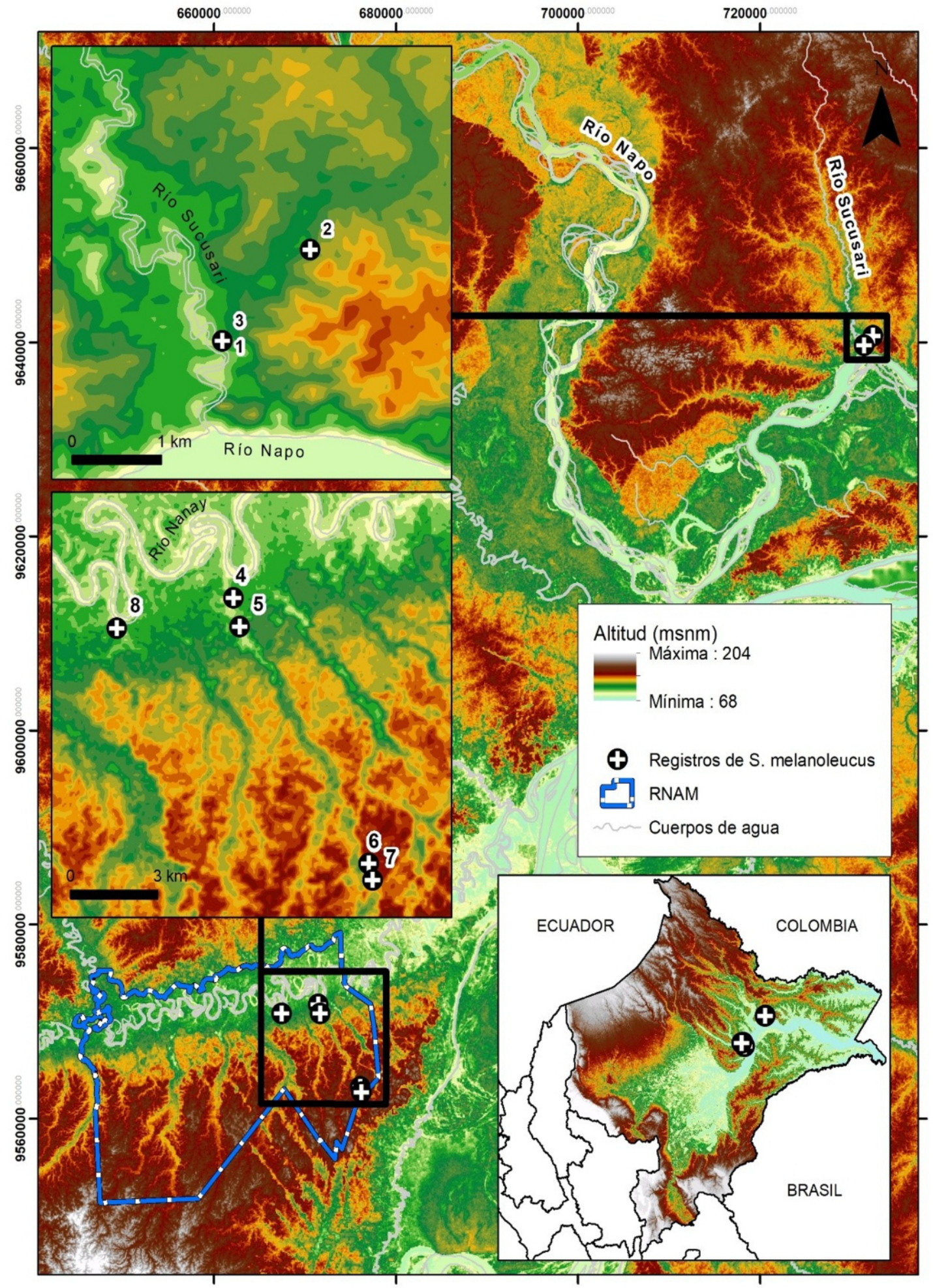

Figura 1. Mapa de registros de Spizaetus melanoleucus en la Reserva Nacional Allpahuayo Mishana y río Sucusari, Loreto, Perú. 


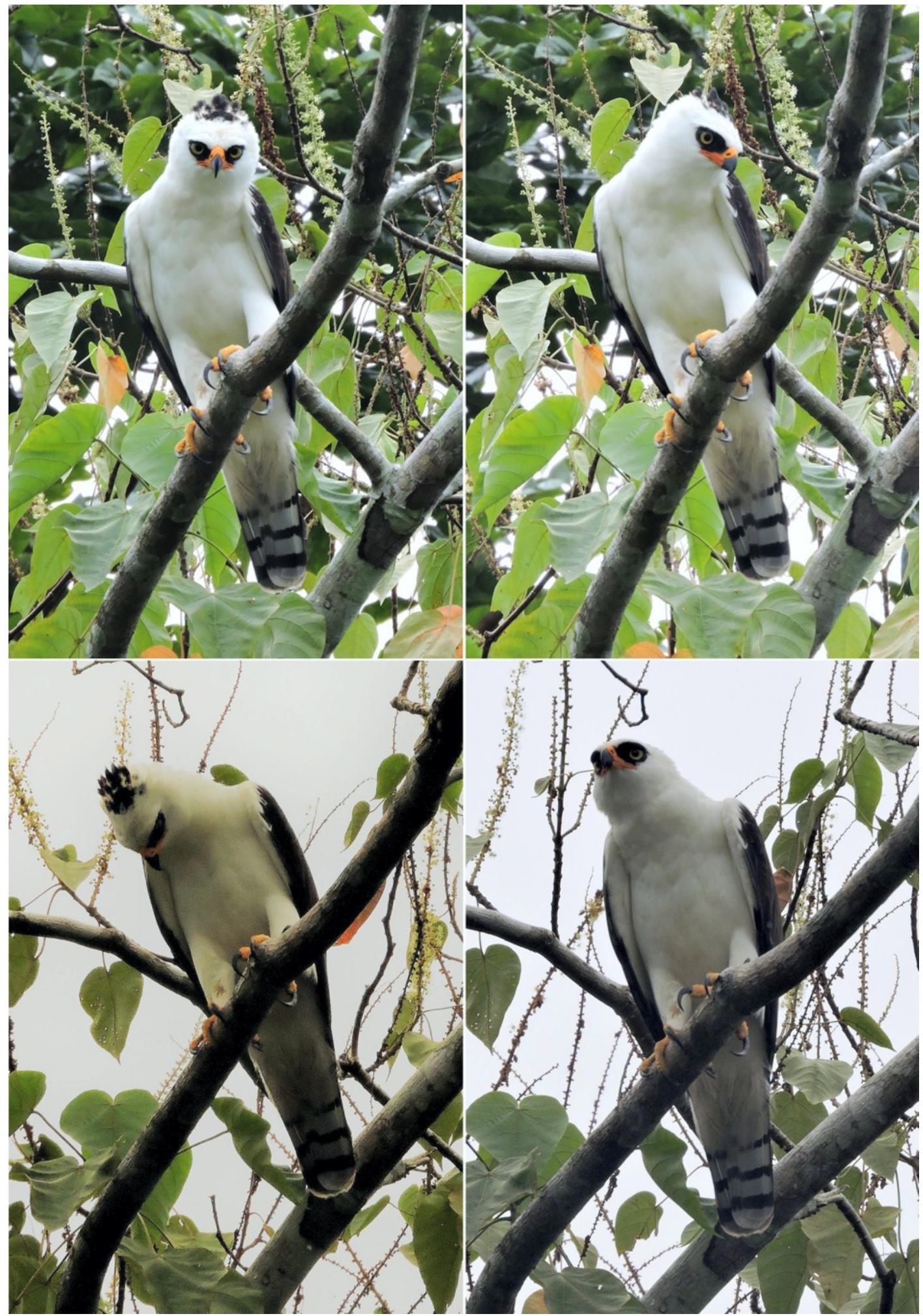

Figura 2. Individuo adulto de Spizaetus melanoleucus registrado en los alrededores de la comunidad de Mishana, Loreto, Perú. Vista frontal (arriba izquierda), vista lateral (arriba derecha), mostrando el detalle del penacho en la cabeza (abajo izquierda) y posición vigilante (abajo derecha). Foto: Jeder Pizango Murrieta. 
El 11 de marzo del 2014, MITV registró a un individuo adulto de Spizaetus melanoleucus en los bosques sobre suelo arcilloso en las parcelas del Instituto Nacional de Innovación Agraria (INIA) a la altura del km 25 de la carretera Iquitos-Nauta (distrito de San Juan, Provincia de Maynas). Posteriormente Thomas Valqui, registró a un individuo adulto el 12 octubre del 2016, en los alrededores del $\mathrm{km} 25$ de la carretera Iquitos-Nauta, en bosques sobre arena blanca en terrenos del INIA. El 12 de marzo del 2017, JPM y MITV registraron y fotografiaron un individuo de Spizaetus melanoleucus, en los alrededores de la comunidad de Mishana (distrito de San Juan, Provincia de Maynas), río Nanay, en el ámbito de la RNAM (Figuras 2-5). La observación del individuo tuvo una duración aproximada de 15 minutos (13:45 a 14:00 h), durante el cual se pudo corroborar que se trababa de un individuo adulto y solitario, perchado a una altura de $20 \mathrm{~m}$ sobre el nivel del suelo, en un árbol conocido localmente como Shamboquiro (Croton palanostigma), muy cerca de la cancha de futbol de la comunidad. El individuo se mostró atento y no emitió ninguna vocalización, probablemente se encontraba acechando a alguna presa, en los alrededores habían muchas colonias de Oropéndolas y Caciques Psarocolius angustifrons y Cacicus cela.

\section{DISCUSIÓN}

El registro documentado del Águila Blanca y Negra Spizaetus melanoleucus en la Reserva Nacional Allpahuayo Mishana constituye el primer reporte de la presencia de esta especie en el área protegida y amplía su rango de distribución hacia el norte del río Amazonas, previamente considerada como escasa o ausente (Schulemberg et al.,2010). Teniendo en cuenta nuestro registro y el reporte de la presencia del migrante boreal Reinita de Connecticut (Oporornis agilis) publicada recientemente (Ungvari-Martin, 2017), el número de especies para la RNAM se estaría incrementando a 498, dos adicionales a las 496 especies de aves reportada (Álvarez et al., 2012).

A pesar de su amplio rango de distribución, esta especie es considerada rara, probablemente debido a su comportamiento silencioso (Hilty \& Brown, 1986; Ridgely \& Greenfield, 2001; Schulenberg et al., 2010). Ocasionalmente puede ser vista perchada en el dosel y/o planeando sobre el bosque de tierra firme, por lo que puede ser confundida fácilmente con otras rapaces de gran tamaño y blanquinegras, como juveniles de Elanio de Cabeza Gris (Leptodon cayanensis), Águila Crestada (Morphnus guianensis) ó el Águila Penachuda (Spizaetus ornatus) (Schulenberg et al., 2010).
Nuestros registros fueron observaciones de individuos adultos solitarios, sin embargo, en Sudamérica existen reportes de desplazamientos de parejas sobrevolando la copa de los árboles (Seipke \& Cabanne, 2002). Nuestras observaciones en áreas abiertas y en áreas cercanas a poblados dentro de la RNAM, coinciden con los hábitats mencionados por otros investigadores (Hilty \& Brown, 1986; Robinson, 1994; Stotz et al., 1996; Ridgely \& Greenfield, 2001). El uso de perchas cercanas a bordes de bosques podría estar relacionado con la búsqueda, detección y captura de especies de aves que son más comunes en áreas abiertas y degradadas, como el Paucar (Cacicus cela), entre otras, ya que forman parte de su dieta (Lehmann, 1943), al igual que pequeños mamíferos y reptiles. Similar comportamiento fue registrado por Robinson (1991) en el Parque Nacional del Manu, aquí reportó a un individuo de Spizaetus melanoleucus capturando individuos del Loro de Cabeza Azúl (Pionus menstruus), mientras consumían arcilla en una colpa. También se menciona el ataque a colonias de Oropéndolas (Psarocolius angustifrons). Más recientemente, Adams \& Williams (2017) reportaron el ataque de Spizaetus melanoleucus a un grupo de Huapos negro cara pelada de Ryland (Pithecia rylandsi) en la amazonia sur del Perú; de igual manera se encontró el esqueleto de un mono del género Callicebus y loros del género Amazona en un nido, en bosques primarios de Tambopata (R. Piana, com. pers.). Durante nuestras observaciones, no registramos ataques a primates, pero en julio del 2013, observamos que los individuos de un grupo de Frailes (Saimiri macrodon) se mostraron notoriamente intranquilos y huyeron ante la presencia de Spizaetus melanoleucus.

Los registros de Spizaetus melanoleucus, considerada una especie rara, nos permite deducir que existen zonas de bosques en la RNAM que se encuentran relativamente en buen estado de conservación desde donde están recolonizando las zonas antrópicas (sistema fuente-sumidero), como en aquellas comunidades del interior de la RNAM: Mishana y San Martin. También es posible que estas comunidades no estén impactando el bosque de manera severa, el cual beneficia a las aves rapaces de gran tamaño, especie con grandes requerimientos territoriales y bajas tasas de reproducción. A pesar de los nuevos reportes para la RNAM que aquí presentamos, es necesario continuar las labores de educación ambiental y el adecuado manejo de los recursos naturales, especialmente de los bosques sobre arena blanca o varillales, bosques inundables y bosques sobre arcilla, que albergan una gran cantidad de presas, y de modo particular los árboles 
de diámetros grandes que probablemente la especie requiere para anidar, especialmente aquellos mayores a 1 m de DAP(R. Piana, com. pers.).

\section{AGRADECIMIENTOS}

A la Jefatura de la Reserva Nacional Allpahuayo Mishana (RNAM-SERNANP) y sus Guardaparques. Especial agradecimiento a los pobladores de la comunidad de Mishana y San Martin por el apoyo logístico brindado. A Pedro Pérez por la elaboración del mapa para el presente reporte y a Tony Mori por la identificación del espécimen botánico. A Van Remsen, Thomas Valqui y Rafael Campos-Ramírez, por permitirnos citar sus registros de Spizaetus melanoleucus. A Manuel A. Plenge por la información bibliográfica proporcionada sobre la especie y a José Álvarez Alonso, Renzo Piana y Fernando Ángulo Pratolongo, por sus invaluables sugerencias al manuscrito.

\section{BIBLIOGRAFÍA}

Adams, B.D.; Williams, M.S. 2017. Fatal attack on Rylands' bald-faced saki monkey (Pithecia rylandsi) by black-and-white haw-eagle (Spizaetus melanoleucus). Primates 58: 368365.

Álvarez, A.J.; Díaz, A.J.; Shany, N. 2012. Avifauna de la Reserva Nacional Allpahuayo Mishana, Loreto, Perú. Cotinga 34: 61-84.

BirdLife International. 2017. Species factsheet: Spizaetus melanoleucus. Accedido el 15 setiembre 2017. <http://datazone.birdlife. org/species/factsheet/Black-and-white-HawkEagle>

eBird. 2017. eBird: An online database of bird distribution and abundance [web application]. eBird, Ithaca, New York. <http://www. ebird.org>.

Ferguson-Lees, J.; Christie, D.A. 2005. Raptors of the World. London, Field Guide. Princeton. New Jersey, USA. 320 pp.
Hilty, S.L.; Brown, W.L. 1986. A Guide to the Birds of Colombia. Princeton University Press, Princeton. New Jersey, USA. 836 PP.

International Union for Conservation of Nature and Natural Resources - IUCN. 2017. The IUCN Red List of Threatened Species. Version 2017-1. Accedido 19 setiembre 2017. <www. iucnredlist.org>

Lehmann. V. F.C. 1943. Notas sobre algunas raras Accipitridas y Falconidas de Colombia. Caldasia. 2 (7):185-190.

Plenge, M.A. 2017. Lista de aves de Perú. Versión 2017. Accedido el 22 de diciembre 2017. $<$ https://sites.google.com/site/boletinunop/chec klist>

Ridgely, S.R.; Greenfield, J.P. 2001. The Birds of Ecuador. Cornell University Press, Ithaca, New York, USA. 740 pp.

Robinson, K.S. 1994. Habitat selection and foraging ecology of raptors in Amazonian Peru. Biotropica 26 (4): 443-458.

Schulenberg, T.S.; Stotz, D.F.; Lane, D.F.; O'Neill, J.P.; Parker III, T.A. 2010. Birds of Peru: revised and updated edition. Princeton University Press, Princeton and Oxford, New Jersey, USA. 664 pp.

Seipke, H.S; Cabanne, S.G. 2002. Rapaces observadas en un área de selvática de San Pedro, Misiones, Argentina. Ornitología Neotropical. 13:223-282.

Ungvari-Martin, J. 2017. Connecticut Warbler (Oporornis agilis) in a Peruvian White Sand Forest Habitat. Boletín UNOP 12(1): 1-3.

Wiley, R.H.; Álvarez, A.J.; Díaz, A.J. 2017. Aves de Loreto Bajo (60-250 m snm) / Birds of lowland Loreto $(60-250 \mathrm{~m}$ asl $)<\mathrm{http}: / /$ www.unc.edu/ rhwiley/loretolist2017/LoretoLits.html>.

Recibido: 31 de Julio del 2017

Aceptado para publicación:30 de Agosto del 2017 